\title{
MS/MS library facilitated MRM quantification of native peptides prepared by denaturing ultrafiltration
}

\author{
Juraj Lenco ${ }^{1,3}$, Renny Lan ${ }^{1}$, Nathan Edwards ${ }^{2}$ and Radoslav Goldman ${ }^{\text {* }}$
}

\begin{abstract}
Naturally occurring native peptides provide important information about physiological states of an organism and its changes in disease conditions but protocols and methods for assessing their abundance are not welldeveloped. In this paper, we describe a simple procedure for the quantification of non-tryptic peptides in body fluids. The workflow includes an enrichment step followed by two-dimensional fractionation of native peptides and MS/MS data management facilitating the design and validation of LC- MRM MS assays. The added value of the workflow is demonstrated in the development of a triplex LC-MRM MS assay used for quantification of peptides potentially associated with the progression of liver disease to hepatocellular carcinoma.
\end{abstract}

Keywords: Native Peptides, denaturing ultrafiltration, MS/MS library, MRM quantitation

\section{Background}

Proteolysis is an important but perhaps the most overlooked eukaryotic post-translational modification. The biology of neuropeptides [1], peptide hormones [2,3], and unusual proteolytically derived signaling molecules $[4,5]$ stimulates interest in the establishment of appropriate analytical workflows. Mass spectrometry is one of the most useful methods for the analysis of complex peptide mixtures. Proteomic assays typically utilize sequence-specific proteases to characterize the components of complex protein mixtures [6] but the methods for analysis of naturally occurring peptides, without a proteolytic step, are less developed. Applications of mass spectrometry to the study of peptides in various body fluids including cerebrospinal fluid [7], urine [8], synovial fluid [9], saliva [10] and of course serum and plasma $[11,12]$ have been described. A universally useful method for the preparation of the peptides for analysis has not yet emerged and context-specific optimization is typically required. The reported methods include ultrafiltration [13], precipitation by organic solvents [14], solid phase extraction [15], size-exclusion chromatography [11], differential solubilization method [16],

\footnotetext{
* Correspondence: rg26@georgetown.edu

'Georgetown University, Department of Oncology, Lombardi Comprehensive Cancer Center, 3970 Reservoir Rd NW, Washington, DC 20057, USA Full list of author information is available at the end of the article
}

and nanoparticle trapping technology [17]. Even methods as simple as direct MALDI-TOF analysis of a complex mixture in a body fluids were used successfully [12] with the benefit of high-throughput, minimal preparative losses of analytes, and minimal sample requirements. Indeed, MALDI- or SELDI-TOF based analyses are most likely the richest source of information about native peptides. On the other hand, these methods suffer from inherent quantitative limitations $[15,18]$. The original semi quantitative screens are therefore followed by the development of isotope dilution kinetic assays [19] and, most recently, multiple reaction monitoring (MRM) LC-MS/MS quantification of target peptides [20].

MRM has emerged as an LC-MS alternative to antibody based assays for accurate protein quantification [21]. This targeted technology for monitoring of select proteins in complex matrices exploits the sensitivity and selectivity of triple quadrupole mass spectrometers. Specific combinations of precursor $\mathrm{m} / \mathrm{z}$ and its fragments (called transitions) are monitored with linear quantification across several orders of magnitude. Perhaps the most valuable feature of the assays is the ability to multiplex hundreds of analytes [22]. The design and validation of new MRM assays, however, is labor and cost intensive if information about the fragmentation of the peptides of interest is not available. For protein 
quantification using a tryptic peptide, empirical data stored in PeptideAtlas may be exploited [23]. PeptideAtlas lists peptides most frequently observed in proteomic studies and their most abundant fragments. Unfortunately, such an information-rich database is not available for native peptides and minimal data applicable to ESI ionization are publically available. This substantially increases the number of potential transitions that must be considered in assay design, to account for multiple precursor charge states, the high number of potential fragments, and their different charge states.

Our overall goal was to develop a workflow leading from standardized preparation of serum samples, through data management facilitating the design of MRM quantification, to the validation in a clinically applicable assay. We have therefore developed and characterized a simple method that efficiently inhibits proteolytic processes in body fluids, with sufficient peptide recovery for LC-MRM MS quantification. To facilitate the design of the MRM assays, we established a Skyline [24] library of MS/MS spectra of native serum peptides. To our best knowledge, this is the first attempt to use 2D-HPLC ESI-MS/MS to build a MS/MS library of native peptides in order to facilitate the design of quantitative MRM assays. As a proof-of-concept, the library was applied to the MRM assay development for three peptides with diagnostic potential in liver cirrhosis and hepatocellular cancer [25-27]. This workflow is not specific to hepatocellular cancer, and is expected to facilitate the development of quantitative LC-MS-MRM assays of native peptides in other studies as well. Specific experimental endpoints may require different preparative steps but we believe that the establishment of a publically available library of MS/MS spectra will facilitate rapid screening of target analytes in clinically relevant samples. This is expected to improve the ability of researchers to validate previously suggested or newly discovered biomarker candidates.

\section{Results and Discussion}

Quantification of peptides in biological samples is of general interest $[2,5,11]$. The mass spectrometric MRM quantification of peptides has become a viable alternative to the traditional ELISA assays [28,29]. MRM quantification is an appealing alternative particularly for multiplex assays of proteolytically modified peptides, such as the native peptides discussed in this article. A discussion of the biology of the native peptides is beyond the scope of this paper; we do not make any claims as to physiological or diagnostic relevance of the peptides analyzed here. We focus on the presentation of a general method for peptide extraction from body fluids and on the generation of native peptide MS/MS library to facilitate the design of quantitative assays for the exploration of the biology of native peptides. It is hard to imagine that a specific quantitative ELISA assay for each of the peptides can be developed; LC-MRM MS is an inviting alternative in this context [28]. We have therefore first optimized a denaturing ultrafiltration method for stabilization and extraction of the native peptides in body fluids and then used the protocol to create a library of MS/MS spectra that helped us optimize efficiently the MRM quantification of three native peptides.

\section{Effect of different solvents on peptide yield}

Achieving stability of peptides in body fluids is a common quantitative challenge $[28,30]$. We have shown by two independent methods that the yield of peptides from serum stabilized with $6 \mathrm{M}$ guanidine hydrochloride (guanidine $\mathrm{HCl} ; \mathrm{G}-\mathrm{HCl}$ in figures) is higher than the yield from serum stabilized with acetonitrile (AcN) (Figure 1). Dilution with water led to about $3 \times$ lower yield compared to guanidine- $\mathrm{HCl}$ while $\mathrm{AcN}$ at $20 \%$ partially improved recovery. This is likely related to proteolytic degradation of the peptides and (non)specific adsorption of native peptides on surfaces and carrier proteins. The highly denaturing guanidine $\mathrm{HCl}$ conditions inactivate trypsin and other proteases and disrupt nonspecific binding of peptides to carrier proteins such as albumin [31] and high-density lipoprotein [32]. Samples containing guanidine $\mathrm{HCl}$ can be efficiently desalted by $\mathrm{C} 18$ solid phase extraction or directly during well-designed reverse phase chromatography. The results suggest that stabilization of the peptides with $6 \mathrm{M}$ guanidine $\mathrm{HCl}$ is more efficient than $20 \%$ AcN.

The ultrafiltration membranes are an efficient enrichment device in combination with the guanidine $\mathrm{HCl}$ denaturation, but it must be pointed out that the cutoff values are not precise; loss of some peptides below the cutoff and leakage of proteins with mass above the cutoff seems unavoidable. The membranes were designed to retain polypeptides above the cutoff; but the recovery of peptides from the filtrates must be verified and optimized. We find that both saturation of non-specific

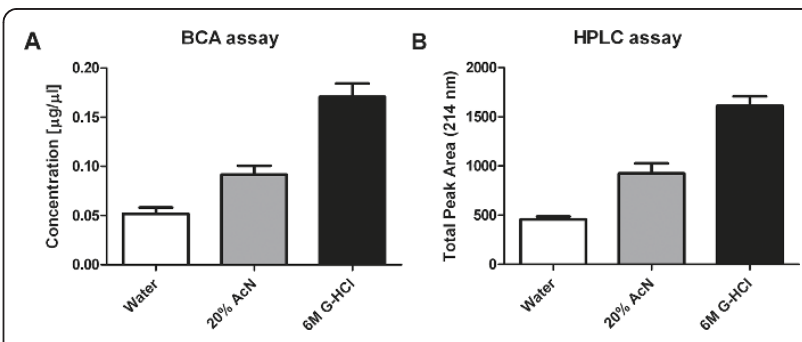

Figure 1 BCA (A) and HPLC-UV (B) quantification of peptides in the ultrafiltrates prepared in water, $20 \%$ Acetonitrile, or $6 \mathrm{M}$ guanidine $\mathbf{H C l}$. Each sample was prepared in triplicate. 
binding sites with carrier proteins such as bovine serum albumin (BSA) and the guanidine $\mathrm{HCl}$ denaturing conditions improve recovery. Other studies have shown that the filtrate may be contaminated by proteins above the mass cut-off of the filters [33]. We have therefore fractionated the ultrafiltrates by HPLC, using a ProSwift RP-1S $4.6 \times 50 \mathrm{~mm}$ monolithic column. Native peptides were detected using MALDI-TOF MS only in early fractions. A standard in-solution tryptic digestion of the later eluting fractions followed by LC-MS/MS identified tryptic peptides of albumin, alpha-2-HS glycoprotein, apolipoprotein A-I and apopolipoprotein C-III. These contaminants eluted from the reverse phase monolith at higher AcN concentrations. Consequently, we used 35\% instead of initial $80 \%$ AcN for elution of peptides from the RP SPE cartridges to further enrich low molecular weight peptides. The chromatograms of ultrafiltrates eluted with $35 \%$ AcN show the removal of the large polypeptide fraction without affecting the recovery of low molecular weight peptides. SDS Bis-Tris gel electrophoresis of proteins further confirmed that $35 \%$ organic SPE elution solvent helped with removal of residual proteins whose bands were detected in the ultrafiltrates eluted from SPE in $80 \%$ AcN. This was accompanied by a decrease of the ultrafiltrate polypeptide concentration measured by BCA assay (Figure 2). The large polypeptides at this low concentration do not directly interfere with the LC-MS analysis of native peptides but can lead to overestimates of the peptide concentration.

\section{Test of denaturing conditions in a BSA/trypsin model and} in serum

To evaluate the inhibition of proteolysis by the guanidine $\mathrm{HCl}$, we used a BSA trypsin digest model. MALDITOF MS analysis demonstrates that guanidine $\mathrm{HCl}$ at a $6 \mathrm{~mol} / \mathrm{l}$ concentration stops the catalytic activity of tryp$\sin$ (Figure 3). The denaturing activity of $20 \% \mathrm{AcN}$, used for this purpose in other studies, including ours [13,14],

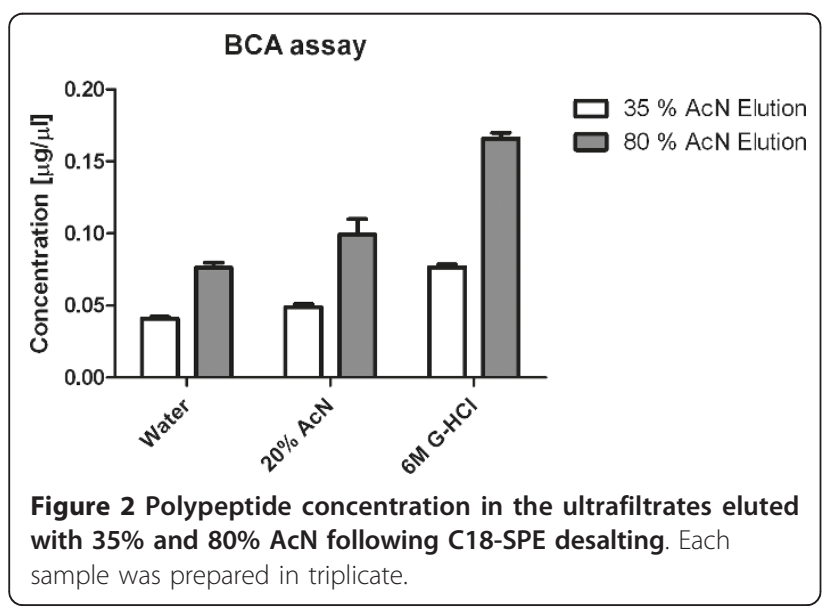

is significantly lower, as trypsin clearly retains activity under these conditions. The concentration of AcN cannot be increased in this setting because higher concentrations are not compatible with the ultrafiltration membrane. The MALDI-TOF results were confirmed by HPLC-UV chromatograms; again, the tryptic activity is similar in the water solvent control and with the addition of $20 \%$ AcN (Figure 3b). Proteolysis is inhibited with $6 \mathrm{M}$ guanidine $\mathrm{HCl}$, where the parent protein remains intact.

To confirm the denaturing efficiency of $6 \mathrm{M}$ guanidine $\mathrm{HCl}$ in serum, we have used heavy analogs of FIBA, $\mathrm{CO} 3, \mathrm{CO} 4$ (fibrinogen alpha chain, complement $\mathrm{C} 3$, and complement $\mathrm{C} 4$ ) native serum peptides observed in our previous studies (Figure 4) [25-27].

The heavy peptide analogs were added to serum right after incubation in either water or guanidine $\mathrm{HCl}$ and the rate of peptide disappearance was evaluated at short $(2 \mathrm{~h})$ and prolonged (overnight) incubation times. The results show substantial degradation of all the tested peptides in serum diluted with water. The peptides have different susceptibility to proteolytic degradation, evidenced by the varying rates of degradation in water at the $2 \mathrm{~h}$ incubation time point. Stabilization of the peptides in serum requires denaturing conditions. The use of $6 \mathrm{M}$ guanidine $\mathrm{HCl}$ stabilizes the peptides quite effectively even at the overnight incubation time. The stabilization of peptides in other body fluids still remains to be verified, but there is no reason to believe that proteolysis would not be inhibited to a similar degree; the $6 \mathrm{M}$ guanidine $\mathrm{HCl}$ should provide effective starting conditions for the optimization of native peptide stabilization and recovery. We suggest that quantification of the native peptides in serum and other body fluids under standardized and validated analytical methods will clarify the controversies reported in the literature.

\section{Recovery of peptides of interest after denaturing ultrafiltration}

Besides the total peptide yield we examined the recovery of three peptides of interest after denaturing ultrafiltration in guanidine $\mathrm{HCl}$. The recovery of $\mathrm{CO} 3$ and $\mathrm{CO} 4$ peptides was $58 \%$ and $54 \%$, FIBA peptide showed greater than $100 \%$ recovery most likely due to its high concentration in the serum that was used as the matrix. We thus repeated the experiment with a BSA solution instead of serum because albumin is the most abundant serum protein and its solution reasonably mimics the serum environment. This resulted in a recovery of $78 \%$.

Identification of native serum peptides using 2D LC-MS/ MS and construction of an MS/MS spectral library

The goal of our study was to design effective LC-MRM MS quantification assays of native peptides. The design 

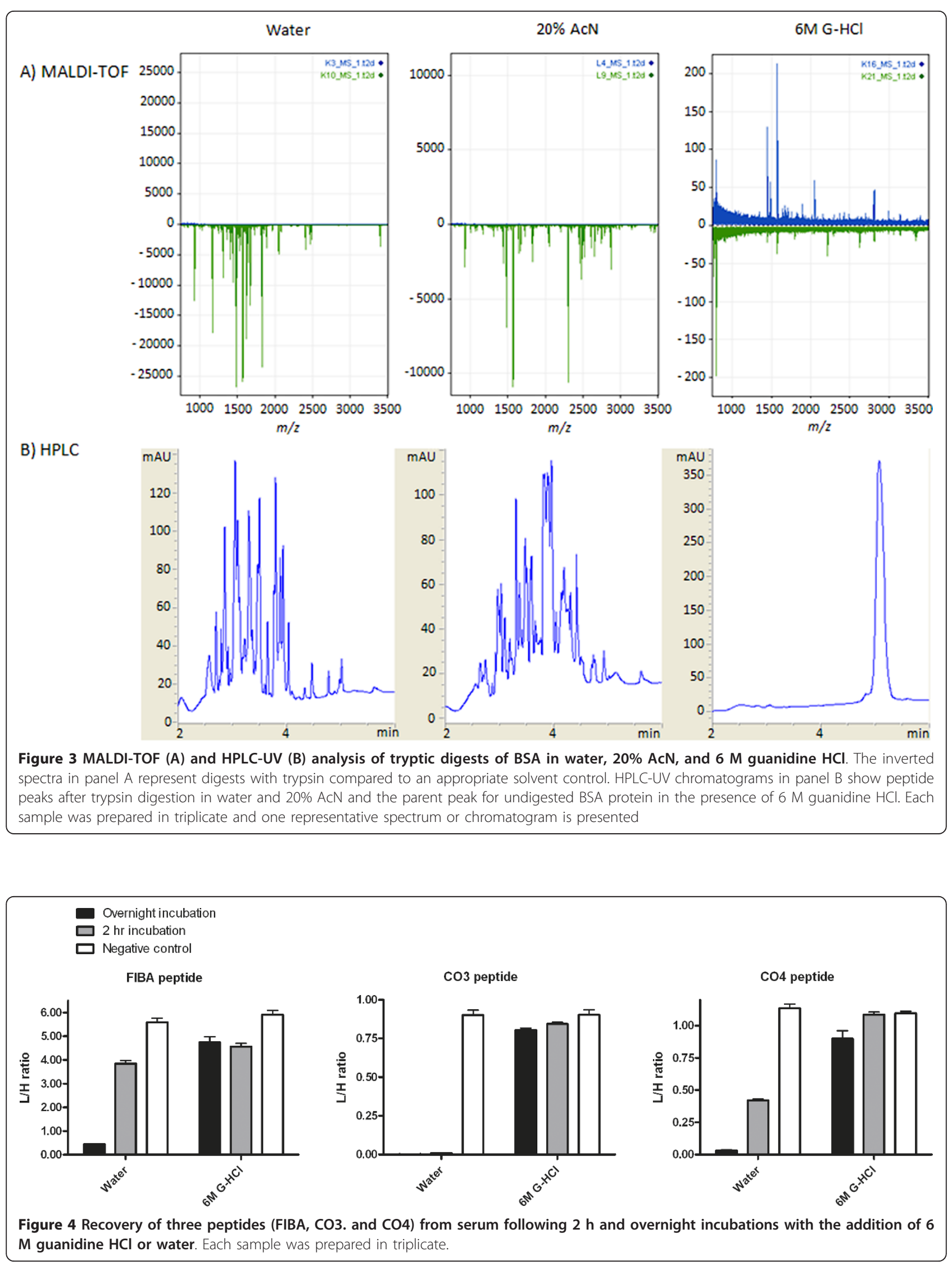


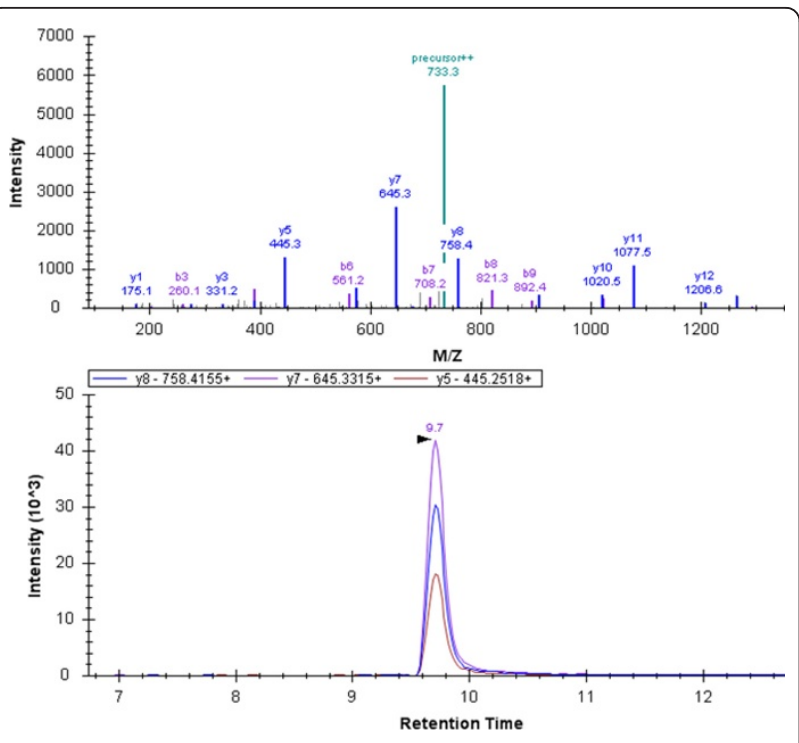

Figure 5 Intensity of fragment ions of the doubly charged FIBA peptide in the MS/MS spectrum; the most intense fragments from the QTOF spectrum correspond to the three most intense 4000 QTRAP MRM transitions.

of assays for the FIBA, CO3, CO4 and other peptides is greatly simplified when information about the likely fragments of the peptides is available. Instead of considering and testing the many possible transitions for each peptide, we decided to build a MS/MS library of spectra to facilitate the design process. To this end, we processed serum samples using the optimized ultrafiltration procedure and fractionated the samples by a two dimensional high/low pH RP chromatography for MS/MS analysis using a QTOF mass spectrometer. The off line high $\mathrm{pH}$ step is orthogonal to the subsequent low $\mathrm{pH}$ nanoLC-MS/MS as described previously and leads to a significant increase in the number of peptide identifications [34]. The combined LC-MS/MS analysis of all the high $\mathrm{pH}$ fractions resulted in the identification of 186 protein groups (at ProteinPilot confidence 95\%) on the basis of 12,762 distinct identified peptides. In order to further reduce the potential for errors in the spectral library, we kept only the highest confidence peptide identifications. After additional filtering of peptide identifications based on precursor mass-delta, reported posttranslational modification, and score, the false discovery rate (FDR) of peptides included in the library was estimated as $1.23 \%$ at the spectra level, $3.61 \%$ at the ion level, and $4.30 \%$ at the peptide level.

Initially, we considered various modified peptides, including deamidated peptides, identified by ProteinPilot for the inclusion in the library; however, our analysis suggests that including these identifications significantly degrades the FDR of the resulting peptide identification set without significantly increasing the number of peptides or ions. The spectra were finally imported into a custom Skyline library (see Additional file 1) in order to create a generally available resource [24]. The final number of precursors imported in the library is 416 , representing 349 unique peptide sequences from 83 proteins (parsimony assignment). Proteins with 10 or more unique native peptides include fibrinogen alpha chain (69), inter-alpha-trypsin inhibitor heavy chain H4 (26), complement C4-A (21), complement C3 (19), fibrinogen beta chain (15), apolipoprotein A-I (12), serum albumin (11), prothrombin (10) and zyxin (10).

The peptide fragmentation in the QTOF (QSTAR Elite, AB Sciex) and 4,000 QTRAP (AB Sciex) mass analyzers are expected to be similar and we find the fragmentation informative for the design of MRM transitions (Figure 5). The QTOF and QTRAP mass spectrometers are both manufactured by AB Sciex; differences in the peptide fragmentation in instruments from other manufactures may be more substantial. The lack of instrument-specific spectra is a significant concern, but long-term, we expect this to be resolved by sharing of MS/MS spectral information by research groups. The importance of PeptideAtlas for the design of MRM assays of tryptic peptides suggests that such as resource for native peptides should be considered [23]. The benefit of the library is perhaps best summarized by the following observation. To design MRM assays for the FIBA, $\mathrm{CO} 3$ and $\mathrm{CO} 4$ peptides, we have considered following peptide characteristics: precursor charges: 2, 3, 4; fragments charges: 1, 2; fragment types: y, b; first fragment - last fragment: 1 to last - $1 ; \mathrm{m} / z$ from - to: 100-2000. A comprehensive testing of peptide fragments with these characteristics requires the consideration of 459 transitions for the CO3, CO4 and FIBA peptides. For tryptic peptides, attention can be focused on y-series fragments for Q3 filter masses, as these are most likely to provide sensitive transitions. This is because trypsin digestion results in peptides with the basic amino acids, arginine or lysine, at the $\mathrm{C}$-terminus, leading to intense $y$-series ions formation under fragmentation. Furthermore, as tryptic peptides are short and usually carry limited number of basic amino acids, doubly charged precursor and singly charged fragments can be preferentially selected for MRM assays. Unfortunately, these heuristic rules cannot be extrapolated to native peptides. This substantially increases the number of potential transitions that must be considered and tested. In fact, for the CO3 peptide SSKITHRIHWESASLL in charge states +3 and +4 , all of the selected transitions were doubly-charged b-ions. The MS/MS library allows us to select just five transitions for each precursor, resulting in the optimization of just 20 transitions. The spectral library turns the task of thoroughly optimizing 459 transitions for the quantification of three peptides into the 
much more reasonable task of checking 20 transitions. Furthermore, without the library, it is unclear when multiply charged or b-ion transitions should be considered, or y-ion transitions removed from consideration. In one case (SSKITHRIHWESASLL), a Lys residue near the Nterminus suggests b-ion transitions should be considered, but for another peptide (NGFKSHALQLNNRQI) with Lys residue near the $\mathrm{N}$-terminus, the Arg residue near the $\mathrm{C}$-terminus ensures that $\mathrm{y}$-ion are abundant. For the peptide DSGEGDFLAEGGGVR, the $\mathrm{y}_{6}$-ion is much less abundant than $y_{7}$ and $y_{5}$ and should perhaps not be used.

\section{MRM analysis}

To demonstrate the utility of the optimized workflow and the MS/MS library, we have designed LC-MRM MS assays for the quantification of the FIBA, CO3, and CO4 peptides in serum samples. These peptides were chosen based on our previous screens, which showed differences in the abundance of these peptides in the serum of hepatocellular cancer patients when compared to the serum of chronic liver disease patients and disease free controls $[25,26]$.

We first selected the five most intense transitions for each peptide based on the data in the MS/MS library. Collision energy was optimized for each transition and the declustering potential was optimized for each precursor. The three most intense transitions per precursor, after optimization, were selected for the FIBA and CO4 peptides; for the $\mathrm{CO} 3$ peptide, the two most intense optimized transitions from both triply and quadruply charged precursors were used (Table 1). We have further tested the linearity of the response for the selected transitions, assessed the potential cross-talk between light and heavy analogues of the peptides, evaluated potential background interference, and estimated the carry-over between chromatographic runs. The assays were linear over 3.4 orders of magnitude with limit of quantification $0.8 \mathrm{fmol} / \mu \mathrm{l}(\mathrm{FIBA}, \mathrm{CO} 4)$ and 4 $\mathrm{fmol} / \mu \mathrm{l}(\mathrm{CO} 3)$ (see). The cross talk was negligible and the carry-over was less than $1 \%$ due to the injection of a blank ( $50 \%$ trifluoroethanol and AcN) between samples. We did not observe a shift in normalized peak areas obtained from spiked heavy labeled peptides or native peptides in serum when compared to the expected values. This shows that the quantification is not affected by background interference. Based on these characteristics, we have adopted these optimized transitions for the absolute quantification of these peptides in serum samples. The optimized assays included denaturation of serum with $6 \mathrm{M}$ guanidine $\mathrm{HCl}$, ultrafiltration, desalting of the filtrate on the C18 SPE cartridges, and a triplex LC-MRM MS quantification as described in the methods. Total run time of the assay was 20 min with the average retention time of $9.78 \mathrm{~min}$ (FIBA), $9.61 \mathrm{~min}$

Table 1 Optimized transitions selected for the quantification of FIBA, CO3, and $\mathrm{CO} 4$ peptides and their heavy analogues

\begin{tabular}{|c|c|c|c|c|c|c|c|c|c|}
\hline Protein name & Peptide sequence & Form & Precursor $m / z$ & Precursor charge & Product $\mathrm{m} / \mathrm{z}$ & Product charge & Fragmention & DP & $\mathrm{CE}$ \\
\hline \multirow[t]{6}{*}{ FIBA } & DSGEGDFLAEGGGVR & Light & 733.33 & 2 & 758.42 & 1 & y8 & 85 & 38.3 \\
\hline & & & 733.33 & 2 & 645.33 & 1 & $y 7$ & 85 & 38.3 \\
\hline & & & 733.33 & 2 & 445.25 & 1 & $\mathrm{y} 5$ & 85 & 38.3 \\
\hline & & Heavy & 735.84 & 2 & 761.43 & 1 & y8 & 85 & 38.3 \\
\hline & & & 735.84 & 2 & 648.34 & 1 & $y 7$ & 85 & 38.3 \\
\hline & & & 735.84 & 2 & 448.26 & 1 & $\mathrm{y} 5$ & 85 & 38.3 \\
\hline \multirow[t]{8}{*}{$\mathrm{CO} 3$} & SSKITHRIHWESASLL & Light & 622.34 & 3 & 810.92 & 2 & b14 & 80 & 27.9 \\
\hline & & & 622.34 & 3 & 867.46 & 2 & b15 & 80 & 26.4 \\
\hline & & Heavy & 624.68 & 3 & 810.92 & 2 & b14 & 80 & 27.9 \\
\hline & & & 624.68 & 3 & 870.97 & 2 & b15 & 80 & 26.4 \\
\hline & & Light & 467.01 & 4 & 767.40 & 2 & b13 & 70 & 21.7 \\
\hline & & & $\overline{467.01}$ & 4 & 810.92 & 2 & b14 & 70 & 17.2 \\
\hline & & Heavy & 468.76 & 4 & 767.40 & 2 & b13 & 70 & 21.7 \\
\hline & & & 468.76 & 4 & 810.92 & 2 & b14 & 70 & 17.2 \\
\hline \multirow[t]{6}{*}{$\mathrm{CO} 4$} & NGFKSHALQLNNRQI & Light & 580.65 & 3 & 885.49 & 1 & $y 7$ & 85 & 29.6 \\
\hline & & & 580.65 & 3 & 757.43 & 1 & y6 & 85 & 31.1 \\
\hline & & & 580.65 & 3 & 644.35 & 1 & $\mathrm{y} 5$ & 85 & 31.1 \\
\hline & & Heavy & 582.99 & 3 & 892.51 & 1 & y7 & 85 & 29.6 \\
\hline & & & 582.99 & 3 & 764.45 & 1 & y6 & 85 & 31.1 \\
\hline & & & 582.99 & 3 & 644.35 & 1 & y5 & 85 & 31.1 \\
\hline
\end{tabular}


(CO3), and 9.32 min (CO4), respectively, for each of the three peptides (Figure 6).The absolute concentration of these peptides, as opposed to relative quantification in our previous studies, were determined in the samples of healthy donors $(n=8)$, patients with chronic liver disease $(n=6)$ and hepatocellular carcinoma (HCC) $(n=$ 7). The heavy labeled peptide standards were spiked into the $8 \mathrm{M}$ guanidine $\mathrm{HCl}$ solution that was added to each serum sample as the first step of the sample preparation. This minimizes the error introduced in absolute quantification that might result from differences in peptide degradation or recovery in the preparative steps.

Kruskal-Wallis test with Dunn's post test confirmed significant differences in concentration of FIBA and CO3 peptides between cancer patients and the healthy and liver disease control groups

The FIBA and CO3 peptides appear significantly lower in the cancer patient samples compared to the chronic liver disease patients and healthy controls as observed in our previous studies. We did not confirm the increase in the $\mathrm{CO} 4$ peptide observed previously; the concentration of this peptide is substantially lower and the variation in measured abundance appears to dominate the magnitude of the change. It is possible that our previous MALDITOF based analysis was not sufficiently accurate, but we cannot, however, exclude other explanations like degradation of the peptide in storage. We want to emphasize that we are not making claims of biological importance or disease classification in this paper; we are presenting a workflow for native peptide extraction and the use of a MS/MS library of spectra for facilitating the design of quantitative MRM assays for native peptides.

\section{Conclusions}

Accurate quantification studies of native peptides are expected to substantially increase our understanding of their function in biological processes. Mass spectrometry offers inviting possibilities for their quantification even in complex biological matrices like body fluids. The semi-quantitative screens based on relative quantification are increasingly being replaced by improved methods such as LC-MRM MS assays. In this paper, we present a workflow for the analysis of native peptides with emphasis on optimization of the enrichment protocol and characterization of the serum peptidome. We demonstrate the benefits of stabilizing serum samples with $6 \mathrm{M}$ guanidine $\mathrm{HCl}$ as well as the added value of the workflow in the facilitated development of LC-MRM MS assay of peptides derived from fibrinogen alpha chain, complement $\mathrm{C} 3$ and complement $\mathrm{C} 4$. The publically available library of MS/MS spectra is expected to facilitate design of the MRM assays by a wider group of investigators. The triplex quantitative method has been evaluated on serum samples of 21 individuals in the context of the progression of liver disease to hepatocellular carcinoma.

\section{Methods \\ Material}

Native peptides derived from fibrinogen alpha chain, complement $\mathrm{C} 3$ and complement $\mathrm{C} 4$ were used in this study. The peptides and their isotopically labeled analogs were purchased from the Synthetic Peptide Application Lab at the University of Pittsburgh (FIBA, DSGEGDFLAEGGGVR) or Emory University Michrochemical Facility (CO3, SSKITHRIHWESASLL and CO4, NGFKSHALQLNNRQI). The DSGEGDFLAEGGGVR peptide analog carried one ${ }^{13} \mathrm{C}$ isotope in each glycine residue, while the SSKITHRIHWESASLL and NGFKSHALQLNNRQI peptides carried six ${ }^{13} \mathrm{C}$ and one ${ }^{15} \mathrm{~N}$ isotopes in a leucine at position 15 and 10 , respectively. Guanidine hydrochloride, trifluoroacetic acid,
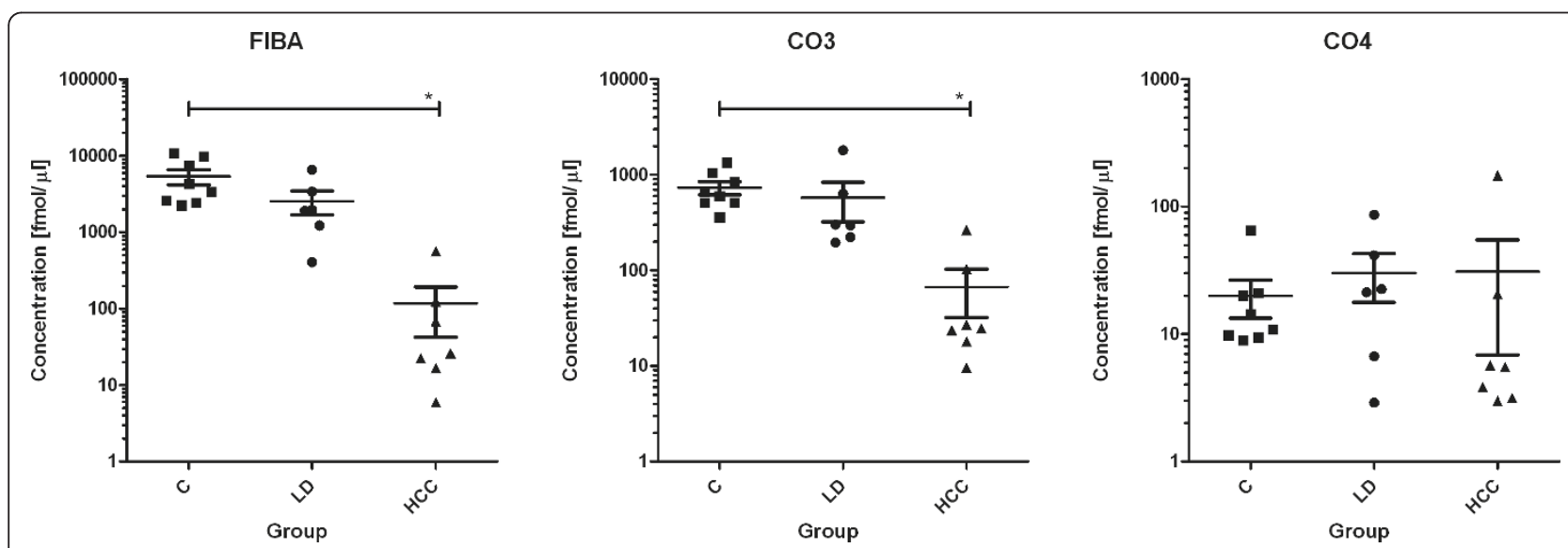

Figure 6 Quantification of FIBA, $\mathrm{CO}$, and $\mathrm{CO} 4$ peptides in samples of healthy controls (C), chronic liver disease patients (LD), and hepatocellular cancer patients (HCC). 
acetonitrile, water, methanol, ${ }^{3} \mathrm{M}$ Empore C18-SD cartridges, and ammonium bicarbonate were purchased from Sigma-Aldrich (St. Louis, MO). BCA assay, BSA standard, methyl methanethiosulfonate, and tris(2-carboxyethyl) phosphine hydrochloride (TCEP) were purchased from Pierce (Rockford, Il). Amicon Ultra-0.5 ml ultrafilters with $30 \mathrm{kDa}$ MWCO membrane were from Millipore (Billerica, MA). Sequencing Grade Modified Trypsin was obtained from Promega (Madison, WI). Water Optima LC/MS, acetonitrile Optima LC/MS, and formic acid (FA) Optima LC/MS were purchased from Fisher Scientific (Fair Lawn, NJ). Guard column XTerra MS C18, 5 $\mu \mathrm{m}, 2.1 \times 20 \mathrm{~mm}$, analytical column XTerra MS $3.5 \mu \mathrm{m}$ C18 $2.1 \times 100 \mathrm{~mm}$, trap column nanoACQUITY UPLC 5 $\mu \mathrm{m}$ Symmetry C18, $180 \mu \mathrm{m} \times 20 \mathrm{~mm}$, and nanoACQUITY UPLC column, $1.7 \mu \mathrm{m}$ BEH C18, $75 \mu \mathrm{m} \times 150$ $\mathrm{mm}$ were obtained from Waters (Milford, MA). Column ProSwift RP-1S, $4.6 \times 50 \mathrm{~mm}$ column was purchased from Dionex (Sunnyvale, CA). PicoTip emitter tips were from New Objective (Woburn, MA). NuPAGE 10\% BisTris electrophoretic gels, running and sample buffers and XCell SureLock Mini-Cell were from Invitrogen (Carlsbad, CA).

\section{Blood collection, processing and serum handling}

Samples were collected in agreement with established IRB protocols. Patients diagnosed with hepatocellular carcinoma were enrolled at the National Cancer Institute of Cairo University, Egypt, from 2000 to 2002 as described previously $[27,35]$. Individuals free of liver disease were recruited from the orthopedic department of Kasr El-Aini Faculty of Medicine at Cairo University and samples from the patients diagnosed with chronic liver disease were acquired at the Ain Shams University Specialized Hospital and the Tropical Medicine Research Institute, Cairo, Egypt as described [27,35]. Briefly, adults (age 18 and older) previously diagnosed with $\mathrm{HCC}$ and those without a prior history of cancer were eligible to participate in the study. The presence of HCC and chronic liver disease was confirmed by pathology, cytology, imaging and elevated levels of serum AFP. Blood samples were collected by a trained phlebotomist each day at around 10 a.m. and processed within a few hours according to a standardized protocol. Aliquots of sera were frozen immediately after collection and stored at $-80^{\circ} \mathrm{C}$ until the time of analysis.

\section{Preparation of native peptides by denaturing ultrafiltration}

Serum $(100 \mu \mathrm{l})$ was diluted with water $(300 \mu \mathrm{l}), 8 \mathrm{M}$ guanidine $\mathrm{HCl}(300 \mu \mathrm{l}$, final concentration $6 \mathrm{~mol} / \mathrm{l})$, or AcN (300 $\mu$ l, final concentration 20\%). The Amicon Ultra-0.5 ml ultrafilters with $30 \mathrm{kDa}$ MWCO membrane were washed twice with $500 \mu \mathrm{l}$ of $6 \mathrm{M}$ guanidine $\mathrm{HCl}$ at
$5.000 \times \mathrm{g}$ for $15 \mathrm{~min}$. The remaining wash solution was removed by converting the filter upside down and spinning at $1.000 \times \mathrm{g}$ for $1 \mathrm{~min}$. Samples were filtered at $8.000 \times \mathrm{g}$ and $30 \mathrm{~min}$. Peptides were cleaned-up using C18 SPE cartridges; 3 M Empore C18-SD cartridges were washed with $0.5 \mathrm{ml}$ of methanol and equilibrated with $0.5 \mathrm{ml}$ of SPE solvent A ( $2 \% \mathrm{AcN}, 0.1 \%$ trifluoroacetic acid (TFA)). After diluting $80 \mu \mathrm{l}$ of the ultrafiltrates with up to $1 \mathrm{ml}$ of the SPE solvent, samples were loaded onto the cartridges and trapped peptides were washed with $1 \mathrm{ml}$ of SPE solvent A. Peptides were eluted with $0.4 \mathrm{ml}$ of SPE solvent B (80\% AcN, $0.1 \%$ TFA or $35 \%$ AcN, $0.1 \%$ TFA) directly into a new micro-tube. BCA assay and a UV-based HPLC assay were used to estimate the peptide yield in the ultrafiltrate. Each sample was prepared for this purpose in triplicate.

\section{BCA assay}

SPE eluates were dried in a vacuum centrifuge and redissolved in $20 \mu \mathrm{l}$ of $0.2 \%$ SDS solution. The BCA assay was used according to the instruction of manufacturer with minor modifications. A $5 \mu \mathrm{l}$ aliquot of each sample was incubated with a $20 \mu \mathrm{l}$ of a 50:1 ratio of the kit reagent $\mathrm{A}: \mathrm{B}$ at $37^{\circ} \mathrm{C}$ for $1 \mathrm{~h}$. Absorbance was measured against a BSA protein standard curve with each sample measured in triplicate. Samples outside the linear range were diluted accordingly. Absorbance at $562 \mathrm{~nm}$ was determined using a micro-volume UV-vis spectrophotometer ND-1000 (Thermo-Fisher, Wilmington DE).

\section{UV-based HPLC assay}

This assay was used for relative comparison of the peptide yield. Fifty microliters of the ultrafiltrate were diluted $20 \times$ by RP solvent A ( $2 \%$ AcN, $0.05 \%$ TFA). For each replicate, $900 \mu \mathrm{l}$ was loaded onto the column on HP 1100 HPLC system (Hewlett Packard, Waldbronn, Germany); we used a guard-column (XTerra MS C18, 5 $\mu \mathrm{m}, 2.1 \times 20 \mathrm{~mm}$ ) heated to $40^{\circ} \mathrm{C}$ at a flow rate of $1 \mathrm{ml} /$ min with the peptides eluted by a step increase from $0 \%$ to $70 \%$ of RP solvent B (98\% AcN, $0.05 \%$ TFA) in 0.01 min. The chromatogram was monitored at $214 \mathrm{~nm}$ and a blank chromatogram resulting from the injection of 10 $\mu \mathrm{l}$ of RP solvent A was subtracted. Area of all detected peaks was summed up as an estimate of peptide quantity. This allowed us to assess the quantity independently of any secondary reaction of the peptides required in the BCA assay.

\section{Recovery of peptides of interest after denaturing ultrafiltration}

Light synthetic peptides FIBA (50 pmol), CO3 (5 pmol) and CO4 $(2 \mathrm{pmol})$ dissolved in $45 \mu \mathrm{l}$ of $8 \mathrm{M}$ guanidine $\mathrm{HCl}$ were added to $15 \mu \mathrm{l}$ of serum. The ultrafiltration was performed as described above. Ultrafiltrate $(30 \mu \mathrm{l})$ 
was spiked with isotopically labeled analogs of FIBA (25 pmol), CO3 (2.5 pmol) and CO4 (1 pmol) and desalted on C18 SPE cartridges as stated above. The concentrated SPE eluate (approximately $50 \mu \mathrm{l}$ ) was mixed with $2.5 \mu \mathrm{l}$ of a matrix solution $(3.6 \mathrm{mg}$ of $\mathrm{CHCA} / \mathrm{ml}$ of $50 \%$ AcN, 0.1\% TFA). From this mixture $0.7 \mu \mathrm{l}$ was spotted in triplicate on an Opti-TOF MALDI sample plate and the MS spectra were recorded in relfectron mode on a 4800 MALDI-TOF/TOF mass spectrometer (Applied Biosystems/MDS Sciex, Foster City, CA). The intensity of peaks corresponding to $\mathrm{m} / z$ of non-labeled and labeled FIBA, $\mathrm{CO} 3$ and $\mathrm{CO} 4$ peptides were extracted and the ratio of light and heavy peptide analogs was calculated. The ratios were compared to ratios obtained from the direct mixture of light and heavy peptides prepared from the same starting solutions that did not undergo ultrafiltration. The recovery was calculated as the change in ratios determined in spiked ultrafiltrates of serum and directly in the peptide mixture.

\section{MALDI-TOF and HPLC-UV analysis of the denaturing conditions in a BSA/trypsin model}

Eight microliters of a BSA solution $(2.5 \mu \mathrm{g} / \mu \mathrm{l})$ was added either to water, $20 \% \mathrm{AcN}$, or $6 \mathrm{M}$ guanidine $\mathrm{HCl}$ as above. BSA was TCEP reduced, blocked by methyl methanethiosulfonate, and digested with $0.4 \mu \mathrm{g}$ of trypsin in $100 \mathrm{mM}$ ammonium bicarbonate buffer, $\mathrm{pH} 7.5$, at $37^{\circ}$ $\mathrm{C}$ for $40 \mathrm{~h}$. Instead of the trypsin solution, water was added as a control. The reaction was stopped with the addition of $380 \mu \mathrm{l}$ of the above RP solvent A. The results were evaluated using MALDI-TOF mass spectrometry and an HPLC analysis with UV detection. For MALDI analysis, $30 \mu \mathrm{l}$ of the sample was desalted on $3 \mathrm{M}$ Empore C18-SD cartridges using the same procedure as described above. The SPE eluates were concentrated in a vacuum centrifuge to approximately $20 \mu \mathrm{l}$. MALDI-TOF analysis was conducted in triplicate as described in the previous section. For the HPLC-UV detection, $200 \mu \mathrm{l}$ was loaded onto a ProSwift RP-1S, $4.6 \times 50 \mathrm{~mm}$ column. The thermostat was set up at $60^{\circ} \mathrm{C}$ and the flow rate was $1.8 \mathrm{ml} /$ min. The gradient was from $0 \%$ to $75 \% \mathrm{~B}$ in $6 \mathrm{~min}$. The chromatogram was monitored at $214 \mathrm{~nm}$.

\section{Test of denaturing potential using three native peptides in serum}

Serum $(15 \mu \mathrm{l})$ was diluted either with $60 \mu \mathrm{l}$ of water or $60 \mu \mathrm{l}$ of $8 \mathrm{M}$ guanidine $\mathrm{HCl}$ (final concentration $6 \mathrm{~mol} /$ 1). Synthetic peptide standards were added to each serum sample as follows to achieve comparable detector response: $187.5 \mathrm{pmol}$ of $\mathrm{CO} 3$ peptide and $37.5 \mathrm{pmol}$ of CO4 peptide. The samples were incubated for two hours and a second batch of samples was incubated overnight. After incubation, isotopically labeled peptides were added to each sample in the following amount: 25 pmol of FIBA, 250 pmol of CO3 and 50 pmol of CO4 which gives initially an approximately 1:1 response (CO3 and CO4) and 6:1 response (FIBA) with respect to the light peptide. Negative control samples were prepared in exactly the same way but without the two hour incubation. Low molecular mass polypeptides including the added synthetic peptides were enriched by ultrafiltration as described above including the C18 SPE cartridge cleanup. MALDI-TOF analysis was conducted in triplicate as described above. The intensity of peaks corresponding to $m / z$ of non-labeled and labeled FIBA, $\mathrm{CO} 3$ and $\mathrm{CO} 4$ peptides were extracted and quantified.

\section{D LC-MS/MS identification of native serum peptides}

Serum $(200 \mu \mathrm{l})$ diluted with $600 \mu \mathrm{l}$ of $8 \mathrm{M}$ guanidine $\mathrm{HCl}$ was kept on ice for $5 \mathrm{~min}$. The remaining steps were as described above without spiking with the standard peptides. For each analysis, we combined two ultrafiltrates. First dimensional HPLC separation was an RP HPLC at pH 10.0. $200 \mathrm{mM} \mathrm{NH}_{4} \mathrm{FA}, \mathrm{pH} \mathrm{10,} \mathrm{was}$ added to the ultrafiltrates to achieve a final concentration of $20 \mathrm{mM} \mathrm{NH}_{4} \mathrm{FA}$. The diluted sample was loaded onto an XTerra MS $3.5 \mu \mathrm{m} \mathrm{C18} 2.1 \times 100 \mathrm{~mm}$ column. The thermostat was set at $40^{\circ} \mathrm{C}$ and the flow rate at 0.2 $\mathrm{ml} / \mathrm{min}$. The peptides were eluted by a $2 \%$ to $62 \% \mathrm{~B}$ in $60 \mathrm{~min}$ formed by basic RP buffer $\mathrm{A}\left(20 \mathrm{mM} \mathrm{NH}_{4} \mathrm{FA}\right.$, $\mathrm{pH} 10)$ and basic RP buffer $\mathrm{B}(90 \% \mathrm{AcN}, 20 \mathrm{mM}$ $\mathrm{NH}_{4} \mathrm{FA}, \mathrm{pH}$ 10). The chromatogram was monitored at $214 \mathrm{~nm}$. Starting at $5 \mathrm{~min}, 14$ fractions were collected each $5 \mathrm{~min}$. Peptides in each fraction were dried and redissolved in $75 \mu \mathrm{l}$ of LC-MS solvent A ( $2 \% \mathrm{AcN}, 0.1 \%$ FA) for an injection onto a nanoACQUITY system (Waters, Milford, MA). Peptides were pre-concentrated on a nanoACQUITY UPLC trapping column, $5 \mu \mathrm{m}$ Symmetry $\mathrm{C}_{18}, 180 \mu \mathrm{m} \times 20 \mathrm{~mm}$ at a flow rate $20 \mu \mathrm{l}$ / min. The peptides were resolved on an analytical nanoACQUITY UPLC column, $1.7 \mu \mathrm{m} \mathrm{BEH} \mathrm{C}_{18}, 75 \mu \mathrm{m}$ $\times 150 \mathrm{~mm}$ by a gradient formed by LC-MS solvents A and $\mathrm{B}(98 \%$ AcN, $0.1 \% \mathrm{FA})$ at the flow rate $300 \mathrm{nl} / \mathrm{min}$. The linear gradient was from $2 \%$ to $40 \%$ B in $33 \mathrm{~min}$ with on-line detection using a Q-TOF tandem mass spectrometer (QSTAR Elite, Applied Biosystems/MDS Sciex, Foster City, CA) equipped with a nanoelectrospray ion source with a PicoTip emitter. The instrument was operated in the Information Dependent Acquisition (IDA) mode in which the MS spectrum was recorded for $1 \mathrm{~s}$ and the 4 strongest $2+, 3+, 4+$ or $5+$ ions were selected for MS/MS with Dynamic Exclusion feature enabled. The MS/MS spectra were recorded in the $\mathrm{m} / z$ range 150 - 2000 using Automatic Collision Energy and Automatic MS/MS Accumulation (max. accumulation was $0.7 \mathrm{~s}$ ). Each sample was injected twice.

The spectral data was searched using ProteinPilot 3.0 (Applied Biosystems/MDS Sciex, Foster City, CA) using 
the Paragon algorithm, which uses a sequence-tag search strategy, facilitating non-specific peptide identification without inflating search times. The search was specified using the following parameters in the Paragon method: sample type - identification; cysteine alkylation - none; digestion -none; instrument - QSTAR Elite ESI, species - Homo sapiens; search effort - thorough. The data were searched against the UniProt protein database. The Proteomics System Performance Evaluation Pipeline incorporated directly in ProteinPilot 3.0 was used to conduct target/decoy searches facilitate initial FDR estimates.

\section{MS/MS spectral library}

ProteinPilot results, including decoy hits, were output in $\mathrm{XML}$ format using the vendor supplied tool, group2xml. The resulting XML file was parsed using an in-house script to permit filtering of the peptide identifications for score and identified post-translational modifications; to remove decoy hit peptides; and to permit re-estimation of peptide, ion, and spectrum level FDR for the remaining identifications. This script was used to eliminate peptide identifications with identification confidence (ProteinPilot Probability) less than 0.95; precursor mass more than $0.2 \mathrm{Da}$ from the theoretical monoisotopic mass or the mass corresponding to the isotope cluster peak associated with one, or two, ${ }^{13} \mathrm{C}$ isotopes; and modifications other than oxidation and Pyro-glu on Glu and Gln. After filtering, the high-quality peptide identifications and their associated tandem mass-spectra, as provided in the XML formatted output of ProteinPilot, were reformatted as mzXML and pepXML, with corresponding faux spectral identifiers. Skyline's spectral library (University of Washington, Seattle, WA) build process reads the mzXML and pepXML file pair and constructs a spectral library suitable for Skyline's MRM design tools. For each peptide ion (distinct peptide sequence, charge and modification state), Skyline clusters repeat identifications and selects a representative spectrum that is, on average, most like the others in the cluster. Only the representative spectra are retained. After ProteinPilot analysis, 28,077 unfiltered peptide identifications were formatted as XML, and 1469 were retained by the above filters. After clustering, the spectral library held MS/MS spectra for 416 peptide ions, representing 349 native peptide sequences, for browsing and MRM assay design. The library, configured for Skyline, facilitates the design of LC-MRM MS assays for native peptides.

\section{LC-MRM MS assays}

Chromatographic separations were performed on a nanoACQUITY system (Waters, Milford, MA). After loading of the samples from a $4^{\circ} \mathrm{C}$ cooled autosampler, peptides were pre-concentrated on a nanoACQUITY UPLC trapping column, $5 \mu \mathrm{m}$ Symmetry C18, $180 \mu \mathrm{m} \times$ $20 \mathrm{~mm}$ at a flow rate $15 \mu \mathrm{l} / \mathrm{min}$ for one min. Fast gradient from $20 \%$ to $45 \%$ of LC-MS solvent B in 5 min at a flow rate $400 \mathrm{nl} / \mathrm{min}$ was used to resolve peptides on an analytical nanoACQUITY UPLC column, $1.7 \mu \mathrm{m} \mathrm{BEH}$ C18, $75 \mu \mathrm{m} \times 150 \mathrm{~mm}$. Peptides were quantified on a 4000 QTRAP hybrid mass spectrometer (AB Sciex) equipped with a nanoelectrospray ion source with a PicoTip emitter. A spray voltage of $2400 \mathrm{~V}$ was used with a source temperature $150^{\circ} \mathrm{C}$. The mass spectrometer was operated in MRM mode with first quadrupole (Q1) filtering with 0.7 and third quadrupole (Q3) filtering with 1.0 unit mass resolution. For all MRM analyses, $30 \mathrm{~ms}$ dwell time was used for each transition. MIDAS based enhanced product ion spectra were recorded with Q1 filter set to 1.0 unit resolution. Enhanced product ion spectra were recorded at a scan speed $4000 \mathrm{amu} / \mathrm{s}$ between 150 to $1200 \mathrm{~m} / \mathrm{z}$, with enabled dynamic fill time and Q0 trapping.

The five most intense transitions for each suitable precursor were selected based on data deposited in the MS/ MS library using Skyline. LC-MRM MS chromatograms were obtained on samples of native peptide from serum. Detected peaks were subsequently validated by a MIDAS experiment. After we unambiguously confirmed the detectability of selected peptides in serum samples, synthetic peptides were used for confirmatory analyses and optimization of collision energy and declustering potential. Three most intense optimized transitions per precursor (FIBA and CO4) were used in the final method for peptide quantification. The confirmatory analyses of synthetic $\mathrm{CO} 3$ peptide revealed that the best intensity transition is produced from its quadruply charged ion. The final method for $\mathrm{CO} 3$ quantification therefore included two most intense optimized transitions from triply and quadruply charged precursors which represent four most intense transition overall. Carryover between injections as well as potential crosstalk between light and heavy equivalents of the peptides was subsequently examined. The final MRM method was examined for the linearity of response and the limit of quantification using relative dilution series experiments (see Additional file 2). Potential background interferences were assessed by comparing normalized peak areas of each precursor to be monitored. The values obtained from injections of heavy labeled peptides dissolved in LC-MS solvent A were compared with those originating from the spiked heavy labeled peptides as well as from the native peptides in the serum samples. The MRM data were processed and evaluated in Skyline.

Samples to be analyzed were prepared according to the protocol described above. Sera $(20 \mu \mathrm{l})$ were mixed 
with $8 \mathrm{M}$ guanidine $\mathrm{HCl}$ containing heavy labeled analogues for absolute quantification. To each microliter of serum, $500 \mathrm{fmol}$ of the FIBA and $\mathrm{CO} 3$ peptides and 50 fmol of the CO4 peptide were added together with 625 fmol of peptide SSKITHRIHWESASLLR per $\mu \mathrm{l}$ of serum, added to decrease non-specific adsorption of the $\mathrm{CO} 3$ peptide. The amount of native peptides enriched from an equivalent of $2 \mu \mathrm{l}$ of original sera was injected for the final analysis and each sample was measured in duplicate. The MRM spectra data files were imported into Skyline for evaluation. The quantity of the peptides was determined by comparison to the spiked internal standard. Data was exported to GraphPad Prism 5 (GraphPad Software, La Jolla, CA) for statistical evaluation.

\section{Additional material}

Additional file 1: MSMS library.blib. Library of MS/MS spectra of serum native peptides that can be readily imported into the Skyline software for MRM transition design.

Additional file 2: Figure 1.pdf. Dilution curves for three native peptides and the respective $C V$ values $(n=3)$.

\section{Abbreviations}

AcN: Acetonitrile; BCA: Bicinchoninic acid assay; BSA: Bovine serum albumin; FA: Formic acid; FDR: False discovery rate; FIBA: Fibrinogen alpha chain; CO3: Complement C3; CO4: Complement C4; guanidine HCl or G-HCl: Guanidine hydrochloride; HCC: Hepatocellular carcinoma; MRM: Multiple reaction monitoring; TCEP: Tris(2-carboxyethyl) phosphine hydrochloride; TFA: Trifluoroacetic acid.

\section{Acknowledgements}

This work was supported by RO1 CA115625 and CA135069 awarded to RG and the CCSG grant NIH P30 CA51008 to the Lombardi Comprehensive Cancer Center supporting the Proteomics and Metabolomics Shared Resource. We would like to thank Dr. Miloslav Sanda for his assistance with the LC-MS/MS analysis of proteins.

\section{Author details}

'Georgetown University, Department of Oncology, Lombardi Comprehensive Cancer Center, 3970 Reservoir Rd NW, Washington, DC 20057, USA. ${ }^{2}$ Georgetown University, Department of Biochemistry and Molecular and Cellular Biology, Washington, DC, USA. ${ }^{3}$ Current address: University Hospital Hradec Kralove; Center for Biomedical Research; Sokolska 581; 50005 Hradec Kralove; Czech Republic.

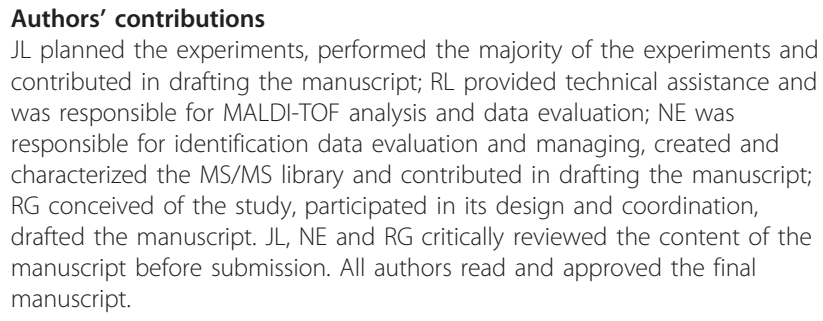
contributed in drafting the manuscript; RL provided technical assistance and was responsible for MALDI-TOF analysis and data evaluation; NE was responsible for identification data evaluation and managing, created and characterized the MS/MS library and contributed in drafting the manuscript; RG conceived of the study, participated in its design and coordination, drafted the manuscript. JL, NE and RG critically reviewed the content of the manuscript before submission. All authors read and approved the final manuscript.

\section{Competing interests}

The authors declare that they have no competing interests.
Received: 26 October 2011 Accepted: 4 February 2012

Published: 4 February 2012

\section{References}

1. Li L, Sweedler JV: Peptides in the brain: mass spectrometry-based measurement approaches and challenges. Annu Rev Anal Chem (Palo Alto (alif) 2008, 1:451-483.

2. Mitchell JW, Atkins N Jr, Sweedler JV, Gillette MU: Direct cellular peptidomics of hypothalamic neurons. Front Neuroendocrinol 2011, 32:377-386.

3. Thevis M, Bredehoft M, Kohler M, Schanzer W: Mass spectrometry-based analysis of IGF-1 and hGH. Handb Exp Pharmacol 2010, 195:201-207.

4. Gomes I, Dale CS, Casten K, Geigner MA, Gozzo FC, Ferro ES, Heimann AS, Devi LA: Hemoglobin-derived peptides as novel type of bioactive signaling molecules. AAPS J 2010, 12:658-669.

5. Keay SK, Szekely Z, Conrads TP, Veenstra TD, Barchi JJ Jr, Zhang CO, Koch KR, Michejda CJ: An antiproliferative factor from interstitial cystitis patients is a frizzled 8 protein-related sialoglycopeptide. Proc Natl Acad Sci USA 2004, 101:11803-11808.

6. McDonald WH, Yates JR III: Shotgun proteomics: integrating technologies to answer biological questions. Curr Opin Mol Ther 2003, 5:302-309.

7. Zougman A, Pilch B, Podtelejnikov A, Kiehntopf M, Schnabel C, Kurnar C, Mann M: Integrated analysis of the cerebrospinal fluid peptidome and proteome. J Proteome Res 2008, 7:386-399.

8. Ling XB, Mellins ED, Sylvester KG, Cohen HJ: Urine peptidomics for clinical biomarker discovery. Adv Clin Chem 2010, 51:181-213.

9. Kamphorst JJ, van der Heijden R, DeGroot J, Lafeber FPJG, Reijmers TH, van Ei B, Tjaden UR, van der Greef J, Hankemeier T: Profiling of endogenous peptides in human synovial fluid by NanoLC-MS: method validation and peptide identification. J Proteome Res 2007, 6:4388-4396.

10. Amado F, Lobo MJC, Domingues P, Duarte JA, Vitorino R: Salivary peptidomics. Expert Rev Proteomics 2010, 7:709-721.

11. Shen Y, Tolic N, Liu T, Zhao R, Petritis BO, Gritsenko MA, Camp DG, Moore RJ, Purvine SO, Esteva FJ, et al: Blood peptidome-degradome profile of breast cancer. PLoS One 2010, 5:e13133.

12. Villanueva J, Lawlor $K$, Toledo-Crow R, Tempst P: Automated serum peptide profiling. Nat Protoc 2006, 1:880-891.

13. Orvisky E, Drake SK, Martin BM, Abdel-Hamid M, Ressom HW, Varghese RS, An $Y$, Saha D, Hortin GL, Loffredo CA, et al: Enrichment of low molecular weight fraction of serum for mass spectrometric analysis of peptides associated with hepatocellular carcinoma. Proteomics 2006, 6:2895-2902.

14. Chertov O, Biragyn A, Kwak LW, Simpson JT, Boronina T, Hoang VM, Prieto DA, Conrads TP, Veenstra TD, Fisher RJ: Organic solvent extraction of proteins and peptides from serum as an effective sample preparation for detection and identification of biomarkers by mass spectrometry. Proteomics 2004, 4:1195-1203.

15. Koomen JM, Li D, Xiao LC, Liu TC, Coombes KR, Abbruzzese J, Kobayashi R: Direct tandem mass spectrometry reveals limitations in protein profiling experiments for plasma biomarker discovery. J Proteome Res 2005, 4:972-981.

16. Kawashima Y, Fukutomi T, Tomonaga T, Takahashi H, Nomura F, Maeda T, Kodera Y: High-yield peptide-extraction method for the discovery of subnanomolar biomarkers from small serum samples. J Proteome Res 2010, 9:1694-1705.

17. Longo C, Patanarut A, George T, Bishop B, Zhou WD, Fredolini C, Ross MM, Espina V, Pellacani G, Petricoin EF, et al: Core-Shell hydrogel particles harvest, concentrate and preserve labile low abundance biomarkers. Plos One 2009, 4:e4763.

18. Davis MT, Auger PL, Patterson SD: Cancer biomarker discovery via low molecular weight serum profiling-are we following circular paths? Physiol Rev 2010, 56:244-247.

19. Villanueva J, Nazarian A, Lawlor K, Yi SS, Robbins RJ, Tempst P: A sequencespecific exopeptidase activity test (SSEAT) for "functional" biomarker discovery. Mol Cell Proteomics 2008, 7:509-518.

20. van den Broek I, Sparidans RW, van Winden AW, Gast MC, van Dulken EJ, Schellens $\mathrm{JH}$, Beijnen $\mathrm{JH}$ : The absolute quantification of eight inter-alphatrypsin inhibitor heavy chain 4 (ITIH4)-derived peptides in serum from breast cancer patients. Proteomics Clin Appl 2010, 4:931-939.

21. Kuhn E, Wu J, Karl J, Liao H, Zolg W, Guild B: Quantification of C-reactive protein in the serum of patients with rheumatoid arthritis using multiple 
reaction monitoring mass spectrometry and 13 C-labeled peptide standards. Proteomics 2004, 4:1175-1186.

22. Anderson NL, Anderson NG, Pearson TW, Borchers CH, Paulovich AG, Patterson SD, Gillette M, Aebersold R, Carr SA: A human proteome detection and quantitation project. Mol Cell Proteomics 2009, 8:883-886.

23. Deutsch EW, Eng JK, Zhang H, King NL, Nesvizhskii Al, Lin B, Lee H, Yi EC, Ossola R, Aebersold R: Human Plasma PeptideAtlas. Proteomics 2005, 5:3497-3500.

24. MacLean B, Tomazela DM, Shulman N, Chambers M, Finney GL, Frewen B, Kern R, Tabb DL, Liebler DC, MacCoss MJ: Skyline: an open source document editor for creating and analyzing targeted proteomics experiments. Bioinformatics 2010, 26:966-968.

25. An Y, Bekesova S, Edwards N, Goldman R: Peptides in low molecular weight fraction of serum associated with hepatocellular carcinoma. Dis Markers 2010, 29:11-20.

26. Goldman R, Ressom HW, Abdel-Hamid M, Goldman L, Wang A, Varghese RS, An Y, Loffredo CA, Drake SK, Eissa SA, et al: Candidate markers for the detection of hepatocellular carcinoma in low-molecular weight fraction of serum. Carcinogenesis 2007, 28:2149-2153.

27. Ressom HW, Varghese RS, Goldman L, An Y, Loffredo CA, Abdel-Hamid M, Kyselova Z, Mechref Y, Novotny M, Drake SK, et al: Analysis of MALDI-TOF mass spectrometry data for discovery of peptide and glycan biomarkers of hepatocellular carcinoma. J Proteome Res 2008, 7:603-610.

28. van den Broek I, Sparidans RW, Schellens JH, Beijnen JH: Quantitative assay for six potential breast cancer biomarker peptides in human serum by liquid chromatography coupled to tandem mass spectrometry. J Chromatogr B Analyt Technol Biomed Life Sci 2010, 878:590-602.

29. Anderson NL, Jackson A, Smith D, Hardie D, Borchers C, Pearson TW: SISCAPA peptide enrichment on magnetic beads using an in-line bead trap device. Mol Cell Proteomics 2009, 8:995-1005.

30. West-Norager M, Kelstrup CD, Schou C, Hogdall EV, Hogdall CK, Heegaard NHH: Unravelling in vitro variables of major importance for the outcome of mass spectrometry-based serum proteomics. J Chromatogr B-Analyt Technol Biomed Life Sci 2007, 847:30-37.

31. Lowenthal MS, Mehta Al, Frogale K, Bandle RW, Araujo RP, Hood BL, Veenstra TD, Conrads TP, Goldsmith P, Fishman D, et al: Analysis of albumin-associated peptides and proteins from ovarian cancer patients. Clin Chem 2005, 51:1933-1945.

32. Hortin GL, Shen RF, Martin BM, Remaley AT: Diverse range of small peptides associated with high-density lipoprotein. Biochem Biophys Res Commun 2006, 340:909-915.

33. Greening DW, Simpson RJ: A centrifugal ultrafiltration strategy for isolating the low-molecular weight $(<=25 \mathrm{~K})$ component of human plasma proteome. J Proteomics 2010, 73:637-648.

34. Gilar M, Olivova P, Daly AE, Gebler JC: Two-dimensional separation of peptides using RP-RP-HPLC system with different $\mathrm{pH}$ in first and second separation dimensions. J Sep Sci 2005, 28:1694-1703.

35. Goldman R, Ressom HW, Varghese RS, Goldman L, Bascug G, Loffredo CA, Abdel-Hamid M, Gouda I, Ezzat S, Kyselova Z, et al: Detection of hepatocellular carcinoma using glycomic analysis. Clin Cancer Res 2009, 15:1808-1813.

doi:10.1186/1477-5956-10-7

Cite this article as: Lenco et al:: MS/MS library facilitated MRM

quantification of native peptides prepared by denaturing ultrafiltration.

Proteome Science 2012 10:7.

\section{Submit your next manuscript to BioMed Central and take full advantage of:}

- Convenient online submission

- Thorough peer review

- No space constraints or color figure charges

- Immediate publication on acceptance

- Inclusion in PubMed, CAS, Scopus and Google Scholar

- Research which is freely available for redistribution

Submit your manuscript at www.biomedcentral.com/submit
Biomed Central 